\title{
The polar wind of the fast rotating Be star Achernar
}

\section{VINCI/VLTI interferometric observations of an elongated polar envelope}

\author{
P. Kervella ${ }^{1}$ and A. Domiciano de Souza ${ }^{2,3}$ \\ 1 LESIA, CNRS UMR 8109, Observatoire de Paris-Meudon, 5 place Jules Janssen, 92195 Meudon Cedex, France \\ e-mail: Pierre.Kervella@obspm.fr \\ ${ }^{2}$ Lab. Univ. d'Astrophysique de Nice (LUAN), CNRS UMR 6525, UNSA, Parc Valrose, 06108 Nice, France \\ 3 Observatoire de la Côte d'Azur, CNRS UMR 6203, Département GEMINI, BP 4229, 06304 Nice Cedex 4, France
}

Received 23 December 2005 / Accepted 20 February 2006

\begin{abstract}
Context. Be stars show evidence of mass loss and circumstellar envelopes (CSE) from UV resonance lines, near-IR excesses, and the presence of episodic hydrogen emission lines. The geometry of these envelopes is still uncertain, although it is often assumed that they are formed by a disk around the stellar equator and a hot polar wind.

Aims. We probe the close environment of the fast rotating Be star Achernar at angular scales of a few milliarcseconds (mas) in the infrared, in order to constrain the geometry of a possible polar CSE.

Methods. We obtained long-baseline interferometric observations of Achernar with the VINCI/VLTI beam combiner in the $H$ and $K$ bands, using various telescope configurations and baseline lengths with a wide azimuthal coverage.

Results. The observed visibility measurements along the polar direction are significantly lower than the visibility function of the photosphere of the star alone, in particular at low spatial frequencies. This points to the presence of an asymmetric diffuse CSE elongated along the polar direction of the star. To our data, we fit a simple model consisting of two components: a 2D elliptical Gaussian superimposed on a uniform ellipse representing the distorted photosphere of the fast rotating star.

Conclusions. We clearly detected a CSE elongated along the polar axis of the star, as well as rotational flattening of the stellar photosphere. For the uniform-ellipse photosphere we derive a major axis of $\theta_{\mathrm{eq}}=2.13 \pm 0.05$ mas and a minor axis of $\theta_{\mathrm{pol}}=$ $1.51 \pm 0.02$ mas. The relative near-IR flux measured for the CSE compared to the stellar photosphere is $f=4.7 \pm 0.3 \%$. Its angular dimensions are loosely constrained by the available data at $\rho_{\text {eq }}=2.7 \pm 1.3$ mas and $\rho_{\text {pol }}=17.6 \pm 4.9$ mas. This CSE could be linked to free-free emission from the radiative pressure driven wind originating from the hot polar caps of the star.
\end{abstract}

Key words. techniques: high angular resolution - techniques: interferometric - stars: emission-line, Be - stars: mass-loss stars: rotation - stars: individual: Achernar

\section{Introduction}

The southern star Achernar ( $\alpha$ Eridani, HD 10144) is the brightest of all Be stars ( $V=0.46 \mathrm{mag}$ ). Depending on the author (and the technique used) the spectral type of Achernar ranges from B3-B4IIIe to B4Ve (e.g., Slettebak 1982; Balona et al. 1987). The estimated projected rotation velocity $v \sin i$ ranges from 220 to $270 \mathrm{~km} \mathrm{~s}^{-1}$ and the effective temperature $T_{\text {eff }}$ from 15000 to $20000 \mathrm{~K}$ (e.g., Vinicius et al. 2006; Rivinius, priv. comm.; Chauville et al. 2001). The difficulty in deriving these parameters more precisely is a direct consequence of the rapid rotation of Achernar. Such rapid rotation ( $\geq 80 \%$ of the critical velocity) induces mainly two effects on the star structure: a rotational flattening and a gravity darkening, which can be described by the von Zeipel effect (von Zeipel 1924).

Domiciano de Souza et al. (2003, hereafter D03) measured the apparent rotational flattening of Achernar using the Very Large Telescope Interferometer (VLTI). They showed that the flattening ratio measured on this star cannot be explained in the Roche approximation, especially when taking the von Zeipel effect into account. Recently, this effect was revealed in two other rapidly rotating stars thanks to interferometric observations: Altair (A7V, Ohishi et al. 2004; Domiciano de Souza et al. 2005) and Regulus (B7V, McAlister et al. 2005).
Rapid rotation and gravity darkening seem to be important keys to explaining the two-component circumstellar environment (CSE) of Be stars: (1) a dense (particle densities $\left.N \simeq 10^{11}-10^{12} \mathrm{~cm}^{-3}\right)$, high mass-loss $\left(\simeq 10^{-8} M_{\odot} / \mathrm{yr}\right)$ and low radial velocity $\left(\simeq 10-100 \mathrm{~km} \mathrm{~s}^{-1}\right)$ equatorial envelope and (2) a rarefied $\left(N \simeq 10^{9} \mathrm{~cm}^{-3}\right)$, low mass-loss $\left(\simeq 10^{-10} M_{\odot} / \mathrm{yr}\right)$ and fast $\left(\simeq 1000 \mathrm{~km} \mathrm{~s}^{-1}\right)$ polar wind (e.g. Damineli Neto \& de Freitas Pacheco 1982; Waters et al. 1987, and references therein). This picture of a two-component CSE is based on many observations of Be stars performed in the past few decades. For example, optical/IR data have shown emission lines and IR excesses that essentially probe the denser regions of the CSE (e.g. Waters 1986; Dougherty et al. 1994), while UV resonance lines of highly ionized species can probe regions of lower density (e.g. Snow 1981; Peters 1982). Gehrz et al. (1974) showed that the near-IR excess measured in Be stars is due to free-free radiation.

The disk-like shape of the dense equatorial CSE has been directly measured by interferometric observations in the radio and optical/IR (e.g. Dougherty \& Taylor 1992; Stee et al. 1995; Quirrenbach et al. 1997). In a recent work, Tycner et al. (2005) explore the relationship between the angular size of the $\mathrm{H} \alpha$ emitting region (measured by interferometry) and the net $\mathrm{H} \alpha$ emission measured spectroscopically for seven Be stars. They find an interesting correlation between the two quantities, which they 
Table 1. Relevant parameters of the calibrators used for VINCI observations of Achernar (continued in Table 2).

\begin{tabular}{lccccccc}
\hline \hline Name & $\alpha$ PsA & $\chi$ Phe & HR 1318 & $\alpha$ Ind & HR 37 & HR 2305 & $\delta$ Phe \\
HD number & HD 216956 & HD 12524 & HD 26846 & HD 196171 & HD 787 & HD 44951 & HD 9362 \\
\hline$m_{\mathrm{V}}$ & 1.2 & 5.2 & 4.9 & 3.1 & 5.3 & 5.2 & 4.0 \\
$m_{\mathrm{K}}$ & 1.0 & 1.3 & 2.3 & 0.9 & 1.8 & 2.3 & 1.7 \\
Sp. type & A3V & K5III & K3III & K0III & K5III & K3III & K0IIIb \\
$T_{\text {eff }}(\mathrm{K})^{a}$ & 8760 & 3780 & 6210 & 4720 & 3780 & 4250 & 4660 \\
$\log g^{a}$ & 4.2 & 1.9 & 2.2 & 2.6 & 1.9 & 2.4 & 2.9 \\
$v \sin i\left(\mathrm{~km} \mathrm{~s}^{-1}\right)^{c}$ & 85 & - & 20 & - & 2 & 20 & - \\
$\theta_{\mathrm{LD}}(\mathrm{mas})^{a}$ & $2.23 \pm 0.07$ & $2.77 \pm 0.03$ & $1.86 \pm 0.02$ & $3.28 \pm 0.03$ & $2.52 \pm 0.03$ & $1.81 \pm 0.03$ & $2.24 \pm 0.02$ \\
$\theta_{\mathrm{UD}}(\mathrm{mas})^{b}$ & $2.19 \pm 0.07$ & $2.69 \pm 0.03$ & $1.81 \pm 0.02$ & $3.20 \pm 0.03$ & $2.45 \pm 0.03$ & $1.76 \pm 0.03$ & $2.18 \pm 0.02$ \\
\hline
\end{tabular}

${ }^{a}$ From Cohen et al. (1999) or Bordé et al. (2002), except $\alpha$ PsA which angular size was measured by Di Folco et al. (2004). ${ }^{b}$ Linear limb darkening coefficients from Claret et al. (1995) or Claret (2000). ${ }^{\mathrm{c}}$ The projected rotational velocities were taken from the catalogue compiled by Glebocki et al. (2000).

attribute to an optically thick emission proportional to the effective area of the emitting disk. Because the equatorial disks are denser $(\simeq 100$ times) than the polar winds and because the free-free emissivity is proportional to the density squared, the equatorial disk dominates the near-IR continuum emission when it is present.

However, it is still not clear if this free-free radiation comes only from the equatorial envelope or if it can also be formed, at least partially, in the polar wind. Modern high angular resolution techniques have the resolving power and sensitivity required to map the spatial distribution of the near-IR emission. In the present paper we investigate this issue by using all available interferometric observations of Achernar obtained with the VINCI/VLTI near-IR instrument (Sect. 2). These observations were performed during a phase where the equatorial disk was nearly absent. The adopted analytical model is presented in Sect. 3 and our results discussed in Sect. 4.

\section{Interferometric observations}

\subsection{Instrumental setup and observations}

The European Southern Observatory's VLTI (Glindemann et al. 2000,2004 ) has been in operation on top of the Cerro Paranal, in Northern Chile since March 2001. For the observations reported in this paper, the light coming from two test siderostats $(0.35 \mathrm{~m}$ aperture) or two Unit Telescopes ( $8 \mathrm{~m}$ aperture) was recombined coherently in VINCI, the VLT INterferometer Commissioning Instrument (Kervella et al. 2000, 2003). We used either a $K$ band $(\lambda=2.0-2.4 \mu \mathrm{m})$ or $H$ band $(\lambda=1.4-1.8 \mu \mathrm{m})$ filter, depending on the beam combiner. In the $K$ band, we relied on the MONA beam combiner, based on fluoride glass optical fibers, while in the $H$ band, we employed the IONIC integrated optics beam combiner (Berger et al. 2001; Kervella et al. 2003; Lebouquin et al. 2004). A total of nine VLTI baselines were used for this program, including five out of the six possible Unit Telescope baselines. Considering the transmission of the instrument and the average effective temperature of Achernar, the effective wavelength of our observations was $\lambda=2.175 \mu \mathrm{m}$ in the $K$ band and $\lambda=1.631 \mu \mathrm{m}$ in the $H$ band. The uncertainty on these wavelengths $(\approx 0.2 \%)$ is negligible compared to the accuracy of our measurements.

\subsection{Data processing and calibration}

The raw data processing was achieved using a wavelet-based algorithm, integrated in an automated data reduction pipeline (Kervella et al. 2004a). The general principle is similar to the original FLUOR algorithm (Coudé du Foresto et al. 1997), but instead of the classical Fourier analysis, we implemented a wavelet-based time-frequency analysis (Ségransan et al. 1999). The output of this pipeline is a single value of the squared coherence factor $\mu^{2}$ for each series of 500 interferograms and the associated bootstrapped error bar. We obtained a total of 49500 interferograms of Achernar in the $K$ band and 9500 in the $H$ band, among which 32394 and 3029 were reduced by the pipeline, respectively. The lower proportion of processed interferograms in the $H$ band is explained by the fact that only one interferometric output is available in the IONIC component, instead of two for the MONA beam combiner. In both cases, two photometric outputs are present. This resulted in a total of 99 squared visibility measurements in the $K$ band, and 19 in the $H$ band, with their associated statistical and calibration uncertainties.

We used a number of calibrators taken mainly from the Bordé et al. (2002) catalogue, which is an adaptation of the Cohen et al. (1999) catalogue for interferometric observations. The observations of these stars were used to estimate the point source response of the interferometer immediately before or after the Achernar observations. Their properties are listed in Tables 1 and 2. The choice of the calibrators is an important step in the preparation of interferometric observations, as significant departures of their actual visibilities from the expected model can propagate into biases on the calibrated visibilities of the scientific target. Among the possible reasons for such departures, binarity (or multiplicity) and deviations from sphericity (due, for instance, to fast rotation or gravitational interaction) are the most critical. All stars in the Bordé et al. (2002) catalogue were carefully scrutinized by these authors for the presence of companions, and are currently regarded as single stars. With respect to fast rotation, the values of $v \sin i$ are generally low for all our calibrators. Spectroscopic measurements of the projected rotational velocities are missing for some of our calibrators; but as they are giant stars, we assume that they are small and, therefore, that the deformation of these stars can be neglected.

One of our calibrators, $\alpha$ PsA (Fomalhaut), is a moderately fast rotating dwarf (A $3 \mathrm{~V}, v \sin i \approx 85 \mathrm{~km} \mathrm{~s}^{-1}$, from Glebocki et al. 2000). We considered carefully the visibilities that were computed using this calibrator, and they show no deviation from the other measurements, in particular those calibrated by $\delta$ Phe that were obtained on the same baseline. Moreover, Di Folco et al. (2004) have measured the angular diameter of this star along the same projected baseline azimuth as during our observations of Achernar. Therefore, we do not expect any difference in terms of angular diameter. In any case, we considered a conservative \pm 0.07 mas $( \pm 3 \%)$ uncertainty on the asumed angular diameter of $\alpha$ PsA. 
Table 2. Relevant parameters of the calibrators used for VINCI observations of Achernar (continued from Table 1). The references are the same as in Table 1.

\begin{tabular}{lccc}
\hline \hline Name & $\epsilon$ Ind A & $\alpha$ Cet & $v$ Cet \\
HD number & HD 209100 & HD 18884 & HD 12274 \\
\hline$m_{\mathrm{V}}$ & 4.7 & 2.5 & 4.0 \\
$m_{\mathrm{K}}$ & 2.2 & -1.7 & 0.0 \\
Sp. type & $\mathrm{K} 4.5 \mathrm{~V}$ & $\mathrm{M} 1.5 \mathrm{III}$ & $\mathrm{K} 5 / \mathrm{M} 0 \mathrm{III}$ \\
$\mathrm{T}_{\text {eff }}(\mathrm{K})$ & 4500 & 3730 & - \\
$\log g$ & 4.5 & - & - \\
$v \sin i\left(\mathrm{~km} \mathrm{~s}^{-1}\right)$ & 1 & - & - \\
$\theta_{\mathrm{LD}}(\mathrm{mas})^{a}$ & $1.89 \pm 0.02$ & - & - \\
$\theta_{\mathrm{UD}}(\mathrm{mas})^{b}$ & $1.84 \pm 0.02$ & $11.6 \pm 0.40$ & $5.3 \pm 0.5$ \\
\hline
\end{tabular}

$a$ The angular diameter of $\epsilon$ Ind $\mathrm{A}$ was measured by Kervella et al. (2004b). ${ }^{b}$ The uniform disk angular sizes of $\alpha$ Cet and $v$ Cet were taken from Dyck et al. (1998) and Richichi \& Percheron (2005), respectively.

The resulting calibrated squared visibilities are listed in Tables $3 \mathrm{a}-\mathrm{d}$. For each measurement, the calibrator is listed. No systematic deviation of the visibility was observed for any of our calibrators at a $1 \sigma$ level.

\section{Model fitting}

\subsection{Polar and equatorial visibilities}

In order to define a plausible model for the light distribution of Achernar, we examine here the shape of the polar and equatorial visibility functions. The orientation of the minor axis of Achernar on the plane of the sky relative to the North was obtained by D03 using a subset of the data discussed in the present paper. Using a simplified analysis of the dependence of the equivalent uniform disk angular diameter with the azimuth of the projected baseline, they obtained an orientation of the minor axis of Achernar (assumed to be the polar axis) of $\alpha_{0}=39 \pm 1^{\circ}$ east of North.

To visualize the polar visibility function of Achernar, we extracted the interferometric measurements with azimuth angles between $10^{\circ}$ and $70^{\circ}$, i.e. $\simeq \pm 30^{\circ}$ from the sky-projected polar axis of the star. As shown in Fig. 2 (left), it appears that the distribution of the measured visibilities does not follow that of a uniform disk, and there is a clear deficit of visibility at low spatial frequencies. Fitting a simple uniform disk model (through a classical least-square minimization) to these data leads to $\theta_{\mathrm{UD}}=1.78 \mathrm{mas}$, but the reduced $\chi^{2}$ of 6.2 is characteristic of a poor fit. The deficit of visibility at low spatial frequencies is typical of the presence of an extended, incoherent source that is already resolved by the interferometer on the short baselines. In other words, a diffuse and extended envelope appears to be present along the polar axis of the star.

The equatorial visibility function can be evaluated by restricting our sample to the visibility measurements obtained in the azimuth range $\alpha_{1}=129 \pm 30^{\circ}$. As shown in Fig. 1, we do not have as many measurements at high spatial frequencies along this range of azimuth, due to the limitations in the available VLTI baselines during commissioning. Figure 2 (right) shows the distribution of squared visibilities observed in this azimuth range as a function of the spatial frequency. In this case, the fit of a simple uniform disk model with $\theta_{\mathrm{UD}}=2.38$ mas produces satisfactory results with a reduced $\chi^{2}$ of only 0.6 . In this case, we conclude that we do not detect any significant diffuse envelope along the equatorial plane of the star.

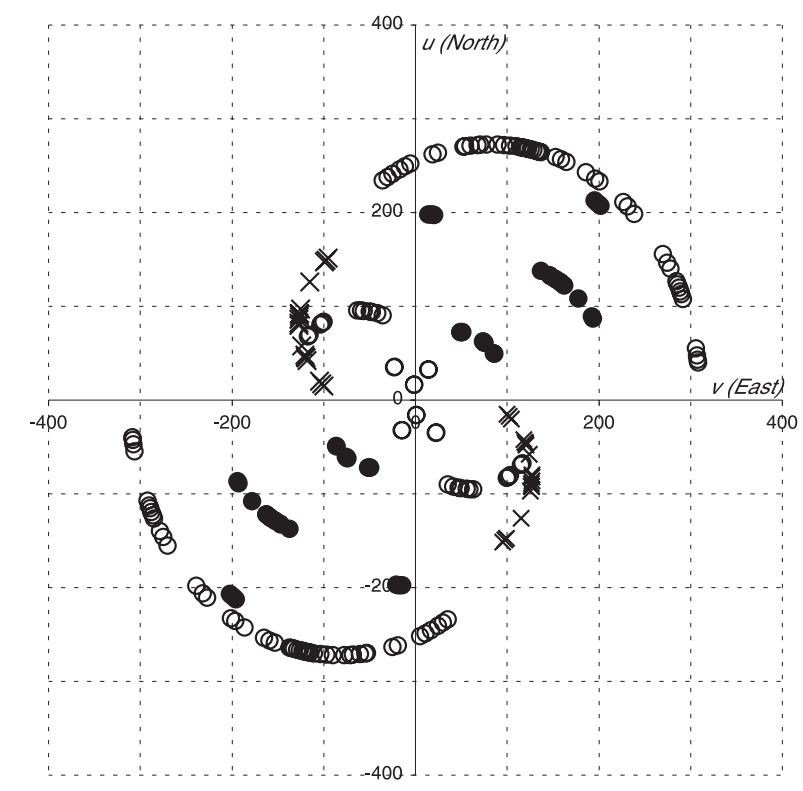

Fig. 1. Coverage of the $(u, v)$ plane for the VINCI observations of Achernar. The $K$ band observations (MONA beam combiner) are represented using circles (open for siderostat observations, solid for the Unit Telescopes), and the $H$ band observations are represented using crosses. The scales are in units of $B / \lambda$, expressed in cycles/arcsec.

\subsection{Star-envelope model description}

As discussed in Sect. 3.1, it appears that a diffuse envelope, confined to the direction of its polar axis, is present around Achernar. In order to study the flux contribution of this envelope, we need to define a simple model to fit the observed visibility data. For this purpose, we considered the following components:

- the stellar photosphere is represented by a uniform ellipse. The parameters are the equatorial and polar angular sizes $\theta_{\mathrm{eq}}$ and $\theta_{\mathrm{pol}}$ and the azimuth orientation of the equatorial axis on the sky $\alpha_{1}$;

- the diffuse envelope is represented by a bidimensional elliptical Gaussian. It is parametrized by its full widths at half maximum (FWHM) along the polar and equatorial axes of the star $\rho_{\mathrm{pol}}$ and $\rho_{\mathrm{eq}}$, and its integrated flux relative to the stellar flux $f$. We make the assumption that its principal axes are aligned with the principal axes of the stellar photosphere.

Using a simple uniform ellipse model is naturally a very simplified approximation of the photospheric light distribution of Achernar. In reality, the rapid rotation of the star causes significant brightening of the polar caps of the star as a consequence of its flattening. Though numerical models can accurately predict the distribution of light on the photosphere of uniformly rotating stars (see e.g. Domiciano de Souza et al. 2002), the underlying Roche approximation is not necessarily verified for Achernar. In particular, Jackson et al. (2004) show that stellar models of Achernar including differential internal rotation result in better agreement with the interferometric profile obtained by D03. Though there are good prospects for differential rotation being constrained observationally by spectro-interferometry (Domiciano de Souza et al. 2004), the current uncertainties on the light distribution of the photosphere lead us to prefer the simple approach of a uniform ellipse. We also make the hypothesis that the axes of the envelope are aligned with the principal axes of the stellar photosphere. Due to the limited coverage of our data set in terms of azimuth angle at intermediate baselines, we 

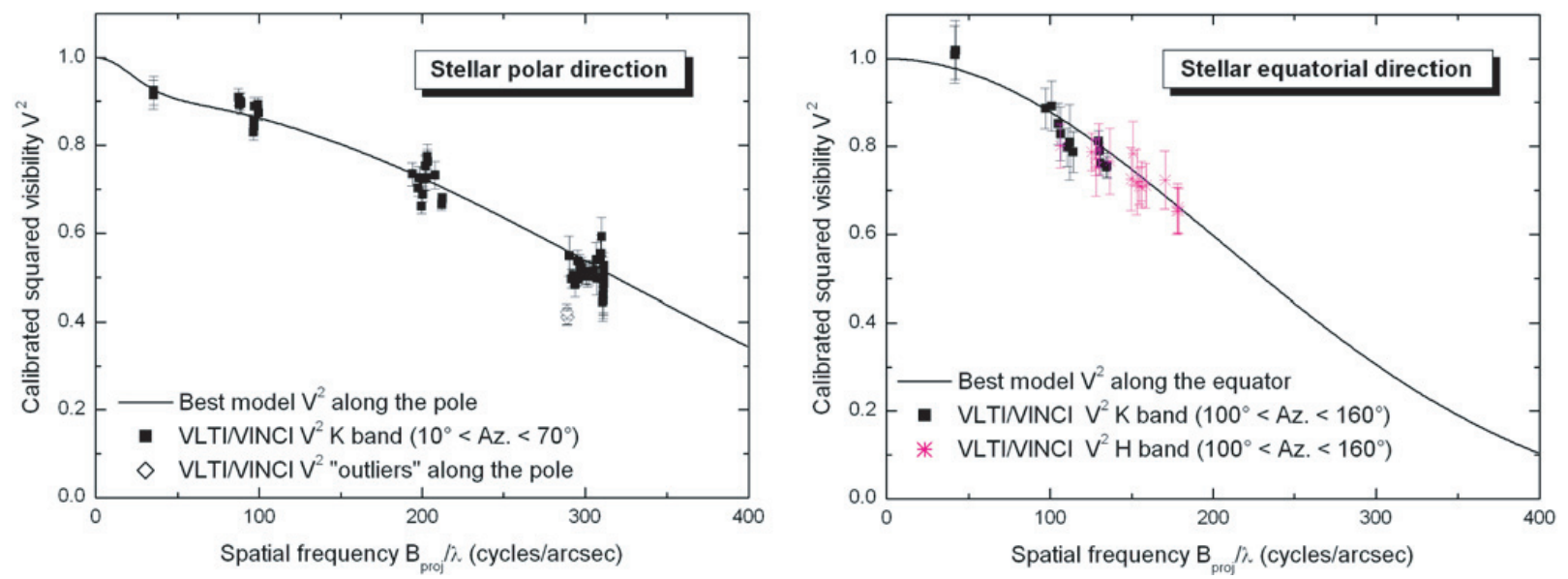

Fig. 2. Left: squared visibilities $V^{2}$ measured on Achernar and corresponding to projected baseline azimuth angles around the polar direction (values between $10^{\circ}$ and $70^{\circ}$ ). Right: $V^{2}$ corresponding to projected baseline azimuth angles around the equatorial direction (values between $100^{\circ}$ and $160^{\circ}$ ). The solid squares and crosses represent $K$ and $H$ band data, respectively. The solid curves represent theoretical $V^{2}$ along the pole $(l e f t)$ and the equator (right) obtained from the 2D fit of our two-component model (Sect. 3) to all measured $V^{2}$ (Tables $3 \mathrm{a}-\mathrm{d}$. Note also the $V^{2}$ outliers along the polar direction, which were not included in the fit (see text for details).

Table 3. (a) Squared visibilities of Achernar in the $K$ band from VINCI, ordered by increasing azimuth angle of the projected baseline. The calibrators are named using their HR number, when no Bayer designation is available. The stated Julian date $\mathrm{JD}_{0}$ is JD $-2.452 \times 10^{6}$. The azimuth is counted in degrees clockwise from North $(N=0 \mathrm{deg}, E=90 \mathrm{deg})$, and $B$ is the projected baseline in meters. The squared visibilities are followed in subscript by the statistical and calibration uncertainties.

\begin{tabular}{|c|c|c|c|c|c|}
\hline $\mathrm{JD}_{0}$ & Cal. & Stations & $B(\mathrm{~m})$ & $\mathrm{Az}$. & $V^{2}(\%)$ \\
\hline 548.551 & $\alpha$ PsA & B3-M0 & 139.44 & 7.3 & $45.7_{ \pm 1.5 \pm 4.1}$ \\
\hline 546.558 & $\alpha$ PsA & B3-M0 & 139.48 & 7.9 & $42.7_{ \pm 4.4 \pm 3.3}$ \\
\hline 548.555 & $\alpha$ PsA & B3-M0 & 139.46 & 8.7 & $46.3_{ \pm 1.5 \pm 4.2}$ \\
\hline 548.560 & $\alpha$ PsA & B3-M0 & 139.48 & 10.2 & $44.4_{ \pm 1.5 \pm 4.0}$ \\
\hline 547.595 & $\alpha$ PsA & B3-M0 & 139.61 & 20.2 & $45.9_{ \pm 2.1 \pm 3.8}$ \\
\hline 545.604 & $\alpha$ PsA & B3-M0 & 139.69 & 21.2 & $50.6_{ \pm 1.6 \pm 2.4}$ \\
\hline 547.600 & $\alpha$ PsA & B3-M0 & 139.63 & 21.8 & $45.0_{ \pm 2.1 \pm 3.7}$ \\
\hline 545.608 & $\alpha$ PsA & B3-M0 & 139.70 & 22.6 & $52.6_{ \pm 1.6 \pm 2.5}$ \\
\hline 547.605 & $\alpha$ PsA & B3-M0 & 139.65 & 23.3 & $46.5_{ \pm 2.2 \pm 3.8}$ \\
\hline 545.613 & $\alpha$ PsA & B3-M0 & 139.72 & 24.0 & $51.7_{ \pm 1.5 \pm 2.5}$ \\
\hline 216.589 & 1318 & U1-U3 & 95.29 & 24.2 & $67.9_{ \pm 1.1 \pm 0.5}$ \\
\hline 216.592 & 1318 & U1-U3 & 95.15 & 24.8 & $66.6_{ \pm 1.3 \pm 0.5}$ \\
\hline 545.622 & $\alpha$ PsA & B3-M0 & 139.72 & 26.7 & $49.7_{ \pm 1.5 \pm 2.4}$ \\
\hline 545.626 & $\alpha$ PsA & B3-M0 & 139.72 & 28.0 & $48.7_{ \pm 2.2 \pm 2.3}$ \\
\hline 534.712 & $\chi$ Phe & U1-U2 & 44.61 & 29.6 & $87.3_{ \pm 1.6 \pm 0.4}$ \\
\hline 545.632 & $\alpha$ PsA & B3-M0 & 139.70 & 29.9 & $51.3_{ \pm 2.2 \pm 2.4}$ \\
\hline 534.714 & $\chi$ Phe & U1-U2 & 44.56 & 30.1 & $89.3_{ \pm 1.6 \pm 0.4}$ \\
\hline 575.637 & 37,2305 & U1-U3 & 93.34 & 31.3 & $73.2_{ \pm 2.8 \pm 1.3}$ \\
\hline 213.654 & $\chi$ Phe & U1-U3 & 91.22 & 36.9 & $76.3_{ \pm 2.2 \pm 1.6}$ \\
\hline 213.655 & $\chi$ Phe & U1-U3 & 91.12 & 37.2 & $77.4_{ \pm 2.3 \pm 1.6}$ \\
\hline 213.658 & $\chi$ Phe & U1-U3 & 90.79 & 37.9 & $72.4_{ \pm 2.2 \pm 1.5}$ \\
\hline 213.660 & $\chi$ Phe & U1-U3 & 90.62 & 38.3 & $75.2_{ \pm 2.0 \pm 1.6}$ \\
\hline 534.749 & $\chi$ Phe & U1-U2 & 43.54 & 38.7 & $88.9_{ \pm 2.1 \pm 0.4}$ \\
\hline 213.663 & $\chi$ Phe & U1-U3 & 90.28 & 39.0 & $72.3_{ \pm 2.0 \pm 1.5}$ \\
\hline 534.750 & $\chi$ Phe & U1-U2 & 43.48 & 39.1 & $84.3_{ \pm 1.5 \pm 0.4}$ \\
\hline 534.752 & $\chi$ Phe & U1-U2 & 43.40 & 39.6 & $85.4_{ \pm 1.6 \pm 0.4}$ \\
\hline 556.635 & $\delta$ Phe & B3-M0 & 139.20 & 39.8 & $59.2_{ \pm 4.1 \pm 1.7}$ \\
\hline 214.664 & 1318 & U1-U3 & 89.89 & 39.9 & $68.8_{ \pm 1.7 \pm 0.5}$ \\
\hline 534.755 & $\chi$ Phe & U1-U2 & 43.32 & 40.2 & $82.9_{ \pm 1.7 \pm 0.4}$ \\
\hline 214.667 & 1318 & U1-U3 & 89.60 & 40.5 & $66.2_{ \pm 1.6 \pm 0.5}$ \\
\hline 556.642 & $\delta$ Phe & B3-M0 & 139.02 & 41.7 & $55.3_{ \pm 4.0 \pm 1.6}$ \\
\hline 213.675 & $\chi$ Phe & U1-U3 & 88.92 & 41.8 & $72.6_{ \pm 2.0 \pm 1.5}$ \\
\hline 214.675 & 1318 & U1-U3 & 88.69 & 42.2 & $70.2_{ \pm 1.7 \pm 0.5}$ \\
\hline 556.646 & $\delta$ Phe & B3-M0 & 138.88 & 42.9 & $54.3_{ \pm 3.8 \pm 1.6}$ \\
\hline 213.691 & $\chi$ Phe & U1-U3 & 87.05 & 45.1 & $73.5_{ \pm 2.2 \pm 1.5}$ \\
\hline
\end{tabular}

Table 3. (b) Squared visibilities of Achernar in the $K$ band from VINCI (continued from Table 3a).

\begin{tabular}{|c|c|c|c|c|c|}
\hline $\mathrm{JD}_{0}$ & Cal. & Stations & $B(\mathrm{~m})$ & Az. & $V^{2}(\%)$ \\
\hline 533.711 & $\epsilon$ Ind & U1-U4 & 129.75 & 45.7 & $41.3_{ \pm 1.2}$ \\
\hline 533.713 & $\epsilon$ Ind & U1-U4 & 129.70 & 46.1 & $41.0_{ \pm 0.9 \pm 1.4}$ \\
\hline 533.715 & $\epsilon$ Ind & U1-U4 & 129.64 & 46.8 & $41.0_{ \pm 0.9 \pm 1.4}$ \\
\hline 533.717 & $\epsilon$ Ind & U1-U4 & 129.57 & 47.4 & $42.4_{+0.9+1.4}$ \\
\hline 550.685 & $\delta$ Phe & B3-M0 & 137.86 & 49.2 & $49.8_{ \pm 3.4 \pm 1.4}$ \\
\hline 550.688 & $\delta$ Phe & B3-M0 & 137.64 & 50.3 & $53.9_{ \pm 3.6 \pm 1.5}$ \\
\hline 550.697 & $\delta$ Phe & B3-M0 & 137.09 & 52.5 & $51.5_{ \pm 1.6 \pm 1.4}$ \\
\hline 534.898 & $\chi$ Phe & U2-U3 & 39.87 & 54.7 & $89.5_{ \pm 2.4 \pm 0.4}$ \\
\hline 534.900 & $\chi$ Phe & U2-U3 & 39.68 & 55.0 & $89.9_{ \pm 1.8 \pm 0.4}$ \\
\hline 534.902 & $\chi$ Phe & U2-U3 & 39.44 & 55.4 & $89.3_{ \pm 1.8 \pm 0.4}$ \\
\hline 534.904 & $\chi$ Phe & U2-U3 & 39.18 & 55.9 & $91.0_{ \pm 1.9 \pm 0.4}$ \\
\hline 556.696 & $\delta$ Phe & B3-M0 & 135.77 & 56.9 & $50.9_{ \pm 2.2 \pm 1.4}$ \\
\hline 556.701 & $\delta$ Phe & B3-M0 & 135.32 & 58.2 & $50.3_{ \pm 2.1 \pm 1.3}$ \\
\hline 556.705 & $\delta$ Phe & B3-M0 & 134.87 & 59.5 & $51.1_{ \pm 2.2 \pm 1.4}$ \\
\hline 550.733 & $\delta$ Phe & B3-M0 & 133.55 & 62.6 & $52.1_{ \pm 2.0 \pm 1.5}$ \\
\hline 590.625 & $\delta$ Phe & B3-M0 & 133.37 & 63.0 & $51.6_{ \pm 0.8 \pm 1.3}$ \\
\hline 550.736 & $\delta$ Phe & B3-M0 & 133.15 & 63.5 & $52.9_{ \pm 1.8 \pm 1.5}$ \\
\hline 590.629 & $\delta$ Phe & B3-M0 & 132.83 & 64.2 & $49.7_{ \pm 0.7 \pm 1.2}$ \\
\hline 550.741 & $\delta$ Phe & B3-M0 & 132.55 & 64.7 & $53.7_{ \pm 1.8 \pm 1.5}$ \\
\hline 569.691 & $\delta$ Phe & B3-M0 & 132.25 & 65.3 & $49.6_{ \pm 1.1 \pm 1.2}$ \\
\hline 945.621 & $\alpha$ PsA & E0-G0 & 15.95 & 65.4 & $92.5_{ \pm 3.2 \pm 0.1}$ \\
\hline 590.634 & $\delta$ Phe & B3-M0 & 132.12 & 65.6 & $49.5_{ \pm 0.8 \pm 1.2}$ \\
\hline 554.735 & $\alpha$ PsA & B3-M0 & 131.85 & 66.1 & $48.4_{ \pm 1.9 \pm 2.0}$ \\
\hline 569.695 & $\delta$ Phe & B3-M0 & 131.58 & 66.6 & $49.7_{ \pm 1.1 \pm 1.2}$ \\
\hline 945.625 & $\alpha$ PsA & E0-G0 & 15.93 & 66.7 & $91.5_{ \pm 3.3 \pm 0.1}$ \\
\hline 590.639 & $\delta$ Phe & B3-M0 & 131.41 & 66.9 & $50.4_{ \pm 0.8 \pm 1.3}$ \\
\hline 569.700 & $\delta$ Phe & B3-M0 & 130.94 & 67.8 & $49.6_{ \pm 1.7 \pm 1.2}$ \\
\hline 579.677 & $\delta$ Phe & B3-M0 & 130.22 & 69.1 & $54.8_{ \pm 4.4 \pm 1.1}$ \\
\hline 579.682 & $\delta$ Phe & M0 & 9.41 & 70.4 & $49.7_{ \pm 4.5 \pm 1.0}$ \\
\hline 579.687 & $\delta$ Phe & & & 71.8 & \\
\hline 555.761 & $\delta$ Phe & B3-M0 & 127.04 & 74.1 & $48.7_{ \pm 1.9 \pm 1.0}$ \\
\hline 555.765 & $\delta$ Phe & B3-M0 & 126.16 & 75.4 & $49.7_{ \pm 3.3 \pm 1.0}$ \\
\hline 577.707 & $\delta$ Phe & B3-M0 & 125.74 & 75.9 & $50.1_{ \pm 3.4 \pm 1.3}$ \\
\hline 577.712 & $\delta$ Phe & B3-M0 & 124.67 & 77.4 & $49.8_{ \pm 3.4 \pm 1.3}$ \\
\hline 577.717 & $\delta$ Phe & B3-M0 & 123.75 & 78.6 & $50.3_{ \pm 3.4 \pm 1.3}$ \\
\hline 552.786 & $\alpha$ PsA & B3-M0 & 123.47 & 79.0 & $49.7_{ \pm 4.2 \pm 3.6}$ \\
\hline 533.781 & $\chi$ Phe & U2-U4 & 89.03 & 84.0 & $64.6_{ \pm 2.9 \pm 1.2}$ \\
\hline 533.783 & $\chi$ Phe & U2-U4 & 88.98 & 84.5 & $64.8_{ \pm 2.5 \pm 1.2}$ \\
\hline 555.798 & $\delta$ Phe & B3-M0 & 118.90 & 84.6 & $49.7_{ \pm 3.5 \pm 1.0}$ \\
\hline
\end{tabular}


Table 3. (c) Squared visibilities of Achernar in the $K$ band from VINCI (continued from Table 3b).

\begin{tabular}{cccrrr}
\hline \hline $\mathrm{JD}_{0}$ & Cal. & Stations & $B(\mathrm{~m})$ & Az. & $V^{2}(\%)$ \\
\hline 533.786 & $\chi$ Phe & U2-U4 & 88.91 & 85.2 & $63.6_{ \pm 2.6 \pm 1.1}$ \\
533.788 & $\chi$ Phe & U2-U4 & 88.83 & 85.8 & $62.9_{ \pm 2.5 \pm 1.1}$ \\
555.802 & $\delta$ Phe & B3-M0 & 117.78 & 85.9 & $50.3_{ \pm 2.8 \pm 1.0}$ \\
579.754 & $\delta$ Phe & B3-M0 & 113.06 & 91.2 & $52.6_{ \pm 4.0 \pm 0.8}$ \\
579.759 & $\delta$ Phe & B3-M0 & 111.82 & 92.6 & $52.0_{ \pm 4.0 \pm 0.8}$ \\
629.580 & $\alpha$ Cet & B3-C3 & 7.37 & 93.1 & $98.2_{ \pm 2.7 \pm 0.0}$ \\
579.763 & $\delta$ Phe & B3-M0 & 110.48 & 94.0 & $52.0_{ \pm 4.3 \pm 0.8}$ \\
629.585 & $\alpha$ Cet & B3-C3 & 7.31 & 94.7 & $102.1_{ \pm 3.4 \pm 0.0}$ \\
544.865 & $\chi$ Phe & B3-M0 & 108.80 & 95.8 & $50.2_{ \pm 3.8 \pm 2.4}$ \\
544.869 & $\alpha$ PsA & B3-M0 & 107.50 & 97.2 & $49.5_{ \pm 3.2 \pm 2.4}$ \\
544.873 & $\alpha$ PsA & B3-M0 & 106.17 & 98.6 & $48.2_{ \pm 3.4 \pm 2.3}$ \\
535.631 & $\chi$ Phe & E0-G1 & 43.57 & 111.5 & $88.7_{ \pm 4.6 \pm 0.5}$ \\
535.642 & $\chi$ Phe & E0-G1 & 45.32 & 114.0 & $89.2_{ \pm 5.7 \pm 0.5}$ \\
538.646 & $\chi$ Phe & E0-G1 & 47.10 & 116.7 & $85.1_{ \pm 4.7 \pm 0.4}$ \\
538.651 & $\chi$ Phe & E0-G1 & 47.74 & 117.7 & $82.9_{ \pm 6.1 \pm 0.4}$ \\
535.673 & $\chi$ Phe & E0-G1 & 49.58 & 120.7 & $79.8_{ \pm 4.3 \pm 0.4}$ \\
535.678 & $\chi$ Phe & E0-G1 & 50.24 & 121.8 & $80.9_{ \pm 8.6 \pm 0.4}$ \\
662.575 & $\alpha$ Cet & E0-G0 & 18.82 & 122.1 & $102.0_{ \pm 4.3 \pm 5.3}$ \\
535.686 & $\chi$ Phe & E0-G1 & 51.24 & 123.6 & $78.7_{ \pm 4.6 \pm 0.4}$ \\
662.579 & $\chi$ Cet & E0-G0 & 18.65 & 123.6 & $100.9_{ \pm 6.0 \pm 2.5}$ \\
528.781 & $\chi$ Phe & E0-G1 & 58.21 & 140.2 & $81.1_{ \pm 2.5 \pm 0.6}$ \\
528.785 & $\chi$ Phe & E0-G1 & 58.49 & 141.1 & $79.0_{ \pm 1.8 \pm 0.6}$ \\
528.790 & $\chi$ Phe & E0-G1 & 58.76 & 142.1 & $76.1_{ \pm 2.4 \pm 0.6}$ \\
528.820 & $\chi$ Phe & E0-G1 & 60.41 & 149.0 & $75.2_{ \pm 2.2 \pm 0.7}$ \\
528.824 & $\chi$ Phe & E0-G1 & 60.60 & 150.0 & $75.7_{ \pm 2.8 \pm 0.7}$ \\
\hline & & & & &
\end{tabular}

Table 3. (d) Squared visibilitiess of Achernar in the $H$ band from VINCI, equipped with the IONIC integrated optics beam combiner.

\begin{tabular}{ccccrc}
\hline \hline $\mathrm{JD}_{0}$ & Cal. & Stations & $B(\mathrm{~m})$ & Az. & $V^{2}(\%)$ \\
\hline 475.861 & $\alpha$ Ind & E0-G1 & 52.48 & 126.0 & $70.8_{ \pm 4.3 \pm 1.6}$ \\
475.870 & $\alpha$ Ind & E0-G1 & 53.39 & 127.8 & $71.1_{ \pm 4.8 \pm 1.6}$ \\
477.781 & $\alpha$ Ind & E0-G1 & 42.26 & 109.7 & $78.7_{ \pm 3.9 \pm 1.7}$ \\
477.785 & $\alpha$ Ind & E0-G1 & 42.98 & 110.7 & $77.4_{ \pm 3.7 \pm 1.7}$ \\
479.941 & $\alpha$ Ind & E0-G1 & 59.78 & 146.1 & $65.3_{ \pm 5.2 \pm 1.4}$ \\
479.944 & $\alpha$ Ind & E0-G1 & 59.95 & 146.8 & $66.0_{ \pm 5.4 \pm 1.4}$ \\
479.949 & $\alpha$ Ind & E0-G1 & 60.19 & 147.9 & $65.3_{ \pm 5.1 \pm 1.4}$ \\
482.791 & $\alpha$ Ind & E0-G1 & 45.98 & 115.0 & $76.6_{ \pm 7.3 \pm 1.7}$ \\
482.824 & $\alpha$ Ind & E0-G1 & 50.39 & 122.1 & $72.5_{ \pm 7.0 \pm 1.6}$ \\
482.827 & $\alpha$ Ind & E0-G1 & 50.80 & 122.8 & $78.4_{ \pm 7.1 \pm 1.8}$ \\
483.718 & $\alpha$ Ind & E0-G1 & 34.05 & 98.7 & $82.0_{ \pm 4.4 \pm 1.8}$ \\
483.722 & $\alpha$ Ind & E0-G1 & 34.83 & 99.7 & $77.5_{ \pm 4.1 \pm 1.7}$ \\
483.727 & $\alpha$ Ind & E0-G1 & 35.78 & 101.0 & $80.1_{ \pm 4.6 \pm 1.7}$ \\
483.831 & $\alpha$ Ind & E0-G1 & 51.56 & 124.2 & $71.9_{ \pm 7.2 \pm 1.7}$ \\
483.835 & $\alpha$ Ind & E0-G1 & 52.05 & 125.1 & $70.9_{ \pm 3.8 \pm 1.7}$ \\
483.839 & $\alpha$ Ind & E0-G1 & 52.51 & 126.0 & $72.0_{ \pm 4.2 \pm 1.7}$ \\
484.767 & $\alpha$ Ind & E0-G1 & 43.17 & 110.9 & $75.3_{ \pm 6.4 \pm 1.4}$ \\
484.771 & $\alpha$ Ind & E0-G1 & 43.72 & 111.7 & $79.3_{ \pm 5.7 \pm 1.4}$ \\
485.887 & $\alpha$ Phe & E0-G1 & 57.36 & 137.5 & $72.3_{ \pm 6.5 \pm 1.2}$ \\
\hline
\end{tabular}

choose this approach in order to reduce the number of fitted parameters, and therefore improve the stability of the convergence of the $\chi^{2}$ minimization.

\subsection{Photospheric visibility function}

The visibility function of a uniform ellipse can be derived from the classical visibility function of a circular uniform disk with an angular diameter $\theta_{\mathrm{UD}}$ :

$V_{\mathrm{UD}}(u, v)=\frac{2 J_{1}(x)}{x}$ where $x=\pi \theta_{\mathrm{UD}} \sqrt{u^{2}+v^{2}}$, with $u$ and $v$ the spatial frequency coordinates in units of $B / \lambda$. In order to obtain the visibility function of the ellipse, we use a rotation of the $(u, v)$ axes and a scaling of the $(u, v)$ variables:

$u^{\prime}=u \cos \alpha_{1}+v \sin \alpha_{1} \quad v^{\prime}=-u \sin \alpha_{1}+v \cos \alpha_{1}$.

The visibility of the uniform ellipse with a major axis $\theta_{\mathrm{eq}}$, a minor axis $\theta_{\mathrm{pol}}$, and a major axis orientation relative to the $u$ axis $\alpha_{1}$ is therefore:

$V_{\mathrm{star}}\left(u, v, \theta_{\mathrm{eq}}, \theta_{\mathrm{pol}}, \alpha_{1}\right)=\frac{2 J_{1}\left(x^{\prime}\right)}{x^{\prime}}$

where $x^{\prime}=\pi \sqrt{\theta_{\mathrm{eq}}^{2} u^{\prime 2}+\theta_{\mathrm{pol}}^{2} v^{\prime 2}}$.

\subsection{Envelope visibility function}

As in Sect. 3.3, we can obtain the visibility function of an elliptical Gaussian brightness distribution from the circularly symmetric case for which we have

$V_{\text {Gauss }}(u, v)=\exp \left[-\frac{\left(\pi \rho \sqrt{u^{2}+v^{2}}\right)^{2}}{4 \ln 2}\right]$

where $\rho$ is the FWHM. In the elliptical case, we therefore obtain

$V_{\mathrm{env}}\left(u, v, \rho_{\mathrm{eq}}, \rho_{\mathrm{pol}}, \alpha_{1}\right)=\exp \left[-\frac{\left(\pi \sqrt{\rho_{\mathrm{eq}}^{2} u^{\prime 2}+\rho_{\mathrm{pol}}^{2} v^{\prime 2}}\right)^{2}}{4 \ln 2}\right]$

with the same expression of $u^{\prime}$ and $v^{\prime}$ as in Sect. 3.3, as we assume that the axes of the Gaussian envelope are aligned with the axes of the photosphere. The indexes "eq" and "pol" refer to the equator and pole of the central star.

\subsection{Extracted parameters}

Combining the visibility expressions presented in Sects. 3.3 and 3.4, we obtain the following expression for our simple model of an elongated ellipse with a superimposed Gaussian envelope:

$V_{\text {model }}\left(u, v, \theta_{\mathrm{eq}}, \theta_{\mathrm{pol}}, \rho_{\mathrm{eq}}, \rho_{\mathrm{pol}}, \alpha_{1}, f\right)=\frac{V_{\mathrm{star}}+f V_{\mathrm{env}}}{1+f}$.

To derive the six free parameters of our model, we proceed through a classical $\chi^{2}$ minimization process, with

$\chi_{\mathrm{tot}}^{2}\left(\theta_{\mathrm{eq}}, \theta_{\mathrm{pol}}, \rho_{\mathrm{eq}}, \rho_{\mathrm{pol}}, \alpha_{1}, f\right)=\sum_{i} \frac{\left[V_{i}^{2}-V_{\text {model }}^{2}\left(u_{i}, v_{i}, \ldots\right)\right]^{2}}{\sigma_{i}^{2}}$

where $V_{i}^{2}$ is one of the VINCI squared-visibility measurements, and $\sigma_{i}^{2}$ its associated total variance. The expression of the reduced $\chi^{2}$ is:

$\chi_{\text {red }}^{2}=\frac{\chi_{\text {tot }}^{2}}{N_{\text {obs }}-\text { d.o.f. }}$

where $N_{\mathrm{obs}}$ is the number of individual observations and d.o.f. $=6$ the numbers of degrees of freedom, considering that we fit a total of six parameters.

The minimum $\chi_{\text {red }}^{2}$ of 0.79 is reached for the parameters listed in Table 4. This low value is characteristic of a good correspondence of our model to the interferometric data. The best-fit 
Table 4. Best fit parameters (and corresponding uncertainties) of our simple model consisting in a Gaussian elliptical envelope superimposed on a uniform ellipse representing the central star. The fit was computed on our complete $H$ and $K$ band data set.

\begin{tabular}{lcl}
\hline \hline$\theta_{\mathrm{eq}}$ & $2.13 \pm 0.05 \mathrm{mas}$ & stellar equatorial angular size \\
$\theta_{\mathrm{pol}}$ & $1.51 \pm 0.02 \mathrm{mas}$ & stellar polar angular size \\
$\alpha_{1}$ & $131.6 \pm 1.4 \mathrm{deg}$ & azimuth of the stellar equator \\
$\rho_{\mathrm{eq}}$ & $2.7 \pm 1.3 \mathrm{mas}$ & envelope FWHM along stellar equator \\
$\rho_{\mathrm{pol}}$ & $17.6 \pm 4.9 \mathrm{mas}$ & envelope FWHM along stellar pole \\
$f$ & $4.7 \pm 0.3 \%$ & relative (envelope to star) near-IR flux \\
\hline
\end{tabular}

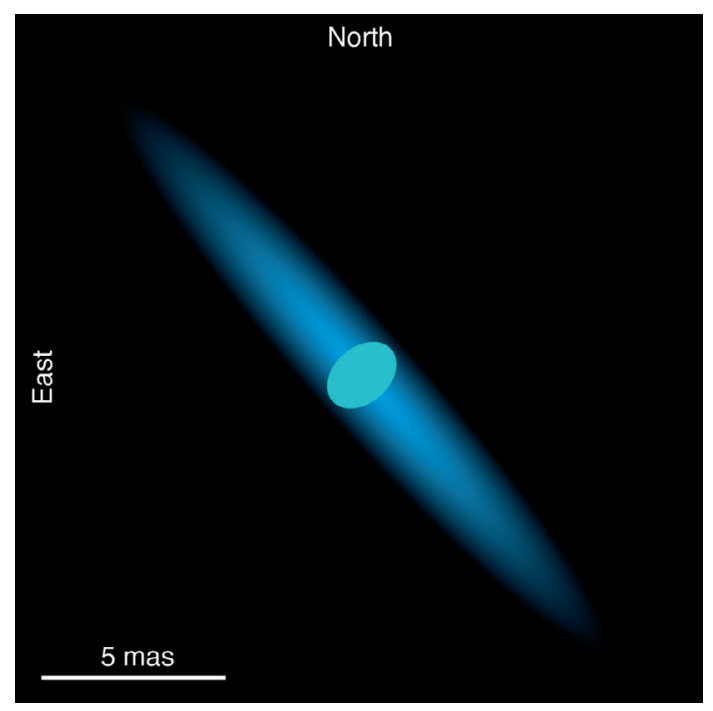

Fig. 3. Graphical representation of the best-fit model intensity distribution of Achernar. The relative flux contributions from the star and the envelope are not to scale. This illustration should not be considered as a true image of the star.

visibility function is a two-dimensional $V^{2}(u, v)$ map. Figure 2 shows the cuts of this best-fit $V^{2}(u, v)$ map along the stellar pole and the equator (solid curves). Note that the rapid visibility decrease observed at low spatial frequencies in the polar direction is reproduced well by the presence of the elongated polar envelope in the model.

A graphical representation of the star and its polar envelope based on the best-fit parameters is presented in Fig. 3. We emphasize that this figure is not a true image of the star, but only the representation of the best-fit light distribution with the a priori hypothesis that the star can be described by a uniform ellipse surrounded by an elliptical Gaussian envelope aligned with its principal axes. This intensity distribution reproduces the observed visibilities well, but several others could also fit. In particular, we cannot determine if the envelope is symmetric relative to the star, due to the baseline orientation ambiguity of $180^{\circ}$.

\subsection{Excluded data points}

In the fitting process, we chose to exclude the four data points obtained on the UT1-UT4 baseline (see Table 3b). With a position angle of $\approx 46 \mathrm{deg}$ for the projected baseline, they correspond to a measurement that is almost aligned with the pole of the star $\left(\alpha_{0}=41.6 \pm 1.4 \mathrm{deg}\right)$. Although they satisfy the data quality criteria that we applied to the other data points, they are located $6-7 \sigma$ away from the best-fit model. It should be noted that the 114 remaining data points are in excellent agreement with our
CSE model, and the residuals of the fit present satisfactory statistical properties (see Sect. 3.7).

An instrumental origin for these outliers cannot be formally excluded, especially as these data points were obtained on the very first night of VLTI operations of the UT1-UT4 baseline. However, no particular technical problem was reported, and the other stars observed on this night showed consistent results. As we could not distinguish these measurements from the rest of our data, we chose to publish them all together for the sake of homogeneity. A possible astrophysical cause for these low visibilities would be a stellar eruption that could have suddenly increased the CSE brightness and/or angular extension.

\subsection{Comparison with other models and residuals of the fit}

In order to assess the level of adequation of our star+CSE model to the data, we also tried to fit them with two simpler models: a circular uniform disk and a uniform ellipse. The residuals for each of the three models are presented in Fig. 4 as a function of the projected baseline azimuth angle. We obtained in the first case a uniform disk angular diameter of $\theta_{\mathrm{UD}}=1.78 \mathrm{mas}$, with the large $\chi_{\text {red }}^{2}$ of 4.9 characteristic of a bad fit. Fitting a uniform ellipse results in the following best-fit values: $\theta_{\mathrm{eq}}=2.31 \mathrm{mas}$, $\theta_{\text {pol }}=1.68$ mas, and $\alpha_{1}=135.7^{\circ}$. Again, the $\chi_{\text {red }}^{2}$ of 3.2 shows poor agreement of this model to our data. It thus appears that our star+Gaussian CSE model is a much better fit to our data set $\left(\chi_{\text {red }}^{2}=0.79\right)$ than the models without CSE.

As shown in Fig. 4 (bottom), the residuals of our star+CSE fit appear to be homogeneous with respect to azimuth angle. Similarly, we do not detect any significant residual either with respect to projected baseline length or with time (Fig. 5). The $H$ and $K$ band data sets do not show any systematic deviation, which justifies a posteriori our combined treatment of these two data sets. Due to the relatively small number of measurements in the $H$ band and their lower accuracy compared to the $K$ band, their influence on the best-fit parameters is very limited. However, they are overall in excellent agreement with the bestfit model, with a specific reduced $\chi^{2}$ of only 0.2 . Considering the limited amount of $H$ band data, we currently cannot investigate the wavelength dependence of the CSE properties, but additional observations with the AMBER instrument of the VLTI in the $J$ and $H$ bands will soon allow such studies. It should be noted that interferometric observations of the bright B0IVpe star $\gamma$ Cas in the visible have shown that the apparent size of this star can vary considerably with wavelength (Stee et al. 1998). The scatter appears to be slightly larger along the polar direction than along the equator of the star (Fig. 5, top). This could be caused by deviations from our simple star-CSE model on small angular scales. For instance, the presence of clumps in the CSE could create this apparent instability of the visibility function. However, our data set is still too limited to constrain their properties significantly.

\section{Nature of the CSE of Achernar}

\subsection{Total extension}

As listed in Table 4, the angular sizes of the axes of the photosphere ellipse $\left(\theta_{\mathrm{eq}}\right.$ and $\left.\theta_{\mathrm{pol}}\right)$ are well constrained, as is the flux ratio $f=4.7 \pm 0.3 \%$ between the star and the polar envelope. However, the angular dimensions of the envelope itself are poorly constrained. In particular, as visible in Fig. 2, we lack very short baseline measurements to estimate the total extension of the envelope in the polar direction. Considering our data, it could be much more extended than the 

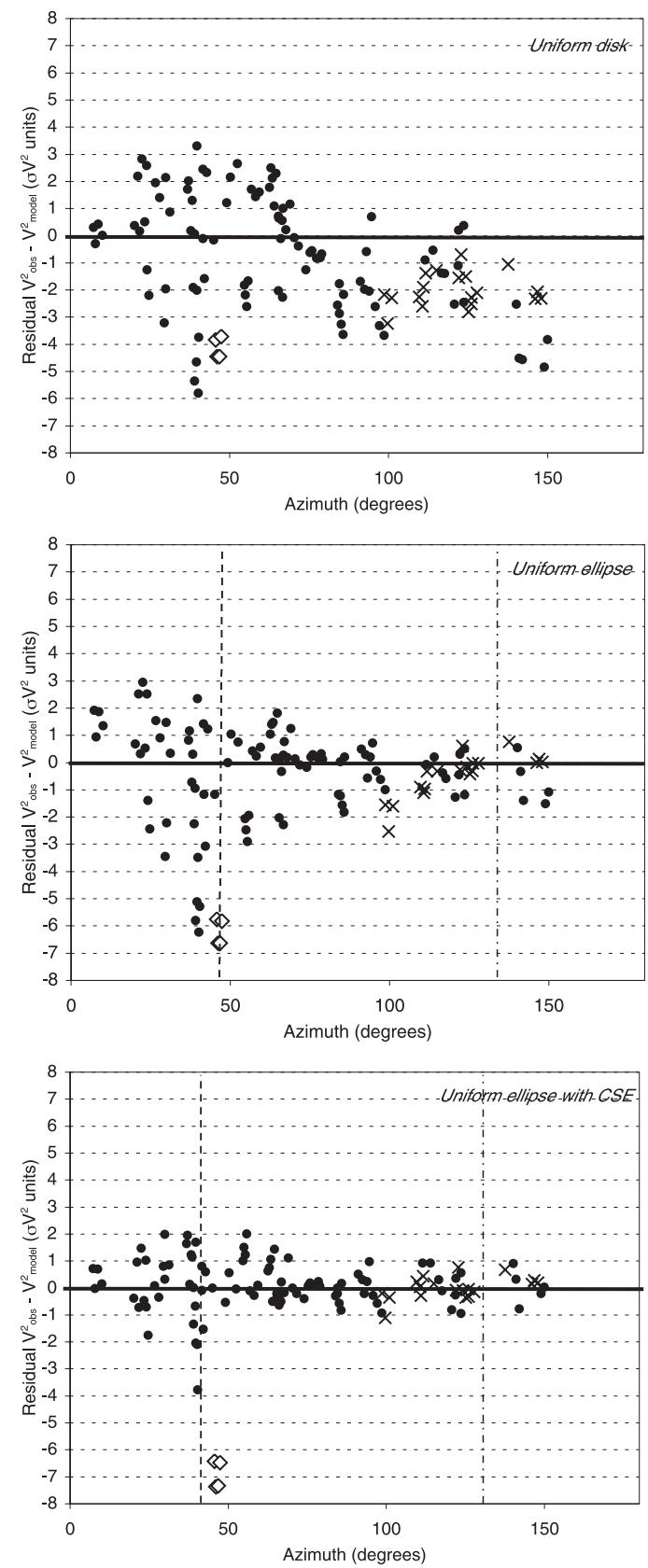

Fig. 4. Residuals of the visibility fit in units of $V^{2}$ standard deviation $\sigma$, as a function of azimuth angle, for a uniform disk (top), a uniform ellipse (middle), and our uniform ellipse with CSE model (bottom). The $H$ band data are shown with crosses. The four outliers (open diamonds) were not included in the fit. The dashed and dot-dashed lines represent, respectively, the polar and equatorial directions of the models, including a uniform ellipse.

derived $\rho_{\text {pol }}=17.6 \pm 4.9$ mas, which should be considered as a lower limit. The angular extension of the envelope in the equatorial direction is also rather poorly constrained by our data, but appears to be small, and could be approximately the size of the star itself.

\subsection{Photospheric flattening ratio}

From the fit of our two-component model, we obtained a photospheric major- over minor-axis ratio of $\theta_{\mathrm{eq}} / \theta_{\mathrm{pol}}=1.41 \pm 0.04$, while D03 measured a value of $1.56 \pm 0.05$, using part of the
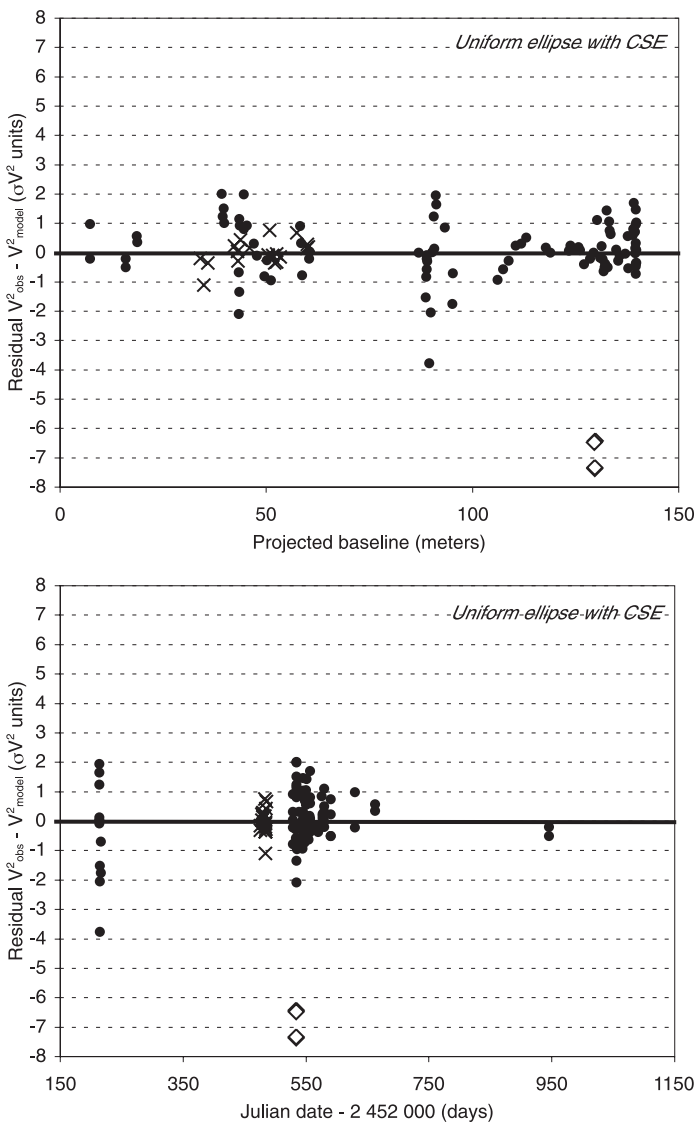

Fig. 5. Residuals of the visibility fit as a function of projected baseline length (top) and date of observation (bottom). The symbols are the same as in Fig. 4.

current data set. The $2.3 \sigma$ difference between these two values can be explained by the difference in adopted model between these two approaches. D03 estimated the uniform disk equivalent angular diameter for each available azimuth and fitted an ellipse on the resulting values. In the present work, we directly fitted our two-component model to the visibilities in the $(u, v)$ plane. In addition, D03 used a single-disk model that does not take the presence of the envelope into account.

Both approaches are valid and have their limitations and advantages. The important point where the main objective concerns the study of the flattening of the star is to compare the results to a physically realistic model including (at least) rotational deformation and gravity darkening, as was done by D03. In a future work we intend to perform a complete astrophysical analysis of the available interferometric and spectroscopic data on Achernar, including rotational effects (flattening and gravity darkening) and the CSE, both in the polar and equatorial directions.

\subsection{Infrared free-free emission}

From the measured flattening ratio, the polar temperature of Achernar could be higher than $20000 \mathrm{~K}$. In this context, the radiation pressure reaches very high values. As was demonstrated in the case of the luminous blue variable star $\eta$ Carinae by Van Boekel et al. (2003), a stellar wind ejected from the poles can have a detectable signature in the interferometric visibilities in the near infrared. Recently, Meilland et al. (2006) showed that an elongated polar wind should be included with 
a thin disk in order to explain the near-IR VLTI/AMBER (e.g., Petrov et al. 2003) observations of $\alpha$ Arae, another Be star that is very similar to Achernar (rotation velocity, spectral type). Although the central stars are similar, one important difference is that $\alpha$ Arae presented hydrogen lines in strong emission during the interferometric observations, while they were absent from the spectrum of Achernar. Both stars show an elongated polar wind responsible for a free-free and free-bound near-IR continuum emission, while only one of them ( $\alpha$ Arae) shows a dense equatorial disk (resolved by VLTI/AMBER) where hydrogen emission lines are formed. This indicates that a significant (in terms of size and near-IR emission) polar wind exists independently if the star is in a normal B or in a Be phase; i.e., the polar wind does not seem to be completely related to the existence of a denser equatorial envelope.

In the hypothesis that the observed polar CSE near-IR emission is mostly caused by free-free radiation, we can roughly estimate the mean electron density as $n_{\mathrm{e}} \simeq 2-3 \times 10^{10} \mathrm{~cm}^{-3}$ for the $H$ and $K$ bands. This value was obtained from the free-free emissivity (e.g. Allen 1973) by considering an electron temperature of $20000 \mathrm{~K}$ (the result does not depend strongly on this value) and by using the CSE parameters derived in this work (Table 4).

In a recent paper, Vinicius et al. (2006) estimated the $2.2 \mu \mathrm{m}$ continuum emission based on a residual emission detected in the $\mathrm{H} \alpha$ absorption profile measured contemporaneously to the VINCI/VLTI campaign on Achernar. They proposed an explanation for the strong flattening measured on Achernar (Domiciano de Souza et al. 2003) by adopting the hypothesis that the residual $\mathrm{H} \alpha$ emission and the associated near-IR continuum emission are formed in the remaining equatorial disk. However, considering the results from the present work, a significant fraction of the near-IR emission appears to originate in the polar envelope.

\section{Conclusion}

We have detected a diffuse circumstellar envelope around the bright Be star Achernar, which accounts for approximately 5\% of the flux of the star in the near-IR ( $H$ and $K$ bands). This envelope presents clear asymmetry with a significantly larger extension along the polar direction of the star. The photosphere of the star itself is distorted by the fast rotation with a larger equatorial angular diameter. The elongation of the CSE points to a significant polar wind, most probably powered by the hot temperature at the stellar poles (von Zeipel effect). Its total extension is loosely constrained by our observations, and it could reach large distances from the star. It appears that a complete astrophysical model able to simultaneously explain all observations (spectroscopic and interferometric) of Achernar is required. We are also confident that spectro-interferometric observations of Achernar with the VLTI/AMBER instrument will bring new insight into the gravity darkening, actual shape and relative intensity of the central star and its immediate circumstellar environment.

Acknowledgements. The interferometric measurements were obtained using the VINCI instrument installed at the VLTI. The VLTI is operated by the European Southern Observatory at Cerro Paranal, Chile. These data were obtained under an unreferenced P70 shared-risk programme and during the technical commissioning of the interferometer. Their processing made use of the waveletbased technique developed by D. Ségransan (Observatoire de Genève) and integrated into the VINCI pipeline. Observations with the VLTI are only made possible through the efforts of the VLTI team, for which we are grateful. The VINCI public commissioning data reported in this paper were retrieved from the ESO/ST-ECF Archive (Garching, Germany). This research made use of the SIMBAD and VIZIER databases at the CDS, Strasbourg (France), and NASA's Astrophysics Data System Bibliographic Services. We thank Drs. O. Chesneau and J. A. de Freitas Pacheco for their enlightening suggestions.

\section{References}

Allen, C. W. 1973, Astrophysical quantities (London: University of London, Athlone Press), 3rd edn.

Balona, L. A., Engelbrecht, C. A., \& Marang, F. 1987, MNRAS, 227, 123

Berger, J.-P., Haguenauer, P., Kern, P., et al. 2001, A\&A, 376, 31

Bordé, P., Coudé du Foresto, V., Chagnon, G., \& Perrin, G. 2002, A\&A, 393, 183

Chauville, J., Zorec, J., Ballereau, D., et al. 2001, A\&A, 378, 861

Claret, A., Diaz-Cordovez, J., \& Gimenez, A. 1995, A\&AS, 114, 247

Claret, A. 2000, A\&A, 363, 1081

Cohen, M., Walker, R. G., Carter, B., et al. 1999, AJ, 117, 1864

Coudé du Foresto, V., Ridgway, S., \& Mariotti, J.-M. 1997, A\&AS, 121, 379

Di Folco, E., Thévenin, F., Kervella, P., et al. 2004, A\&A, 426, 601

Damineli Neto, A., \& de Freitas-Pacheco, J. A. 1982, MNRAS, 198, 659

Domiciano de Souza, A., Vakili, F., Jankov, S., Janot-Pacheco, E., \& Abe, L. 2002, A\&A, 393, 345

Domiciano de Souza, A., Kervella, P., Jankov, S., et al. 2003, A\&A, 407, L47

Domiciano de Souza, A., Zorec, J., Jankov, S., et al. 2004, A\&A, 418, 781

Domiciano de Souza, A., Kervella, P., Jankov, S., et al. 2005, A\&A, 442, 567

Dougherty, S. M., \& Taylor, A. R. 1992, Nature, 359, 808

Dougherty, S. M., Waters, L. B. F. M., Burki, G., et al. 1994, A\&A, 290, 609

Dyck, H. M., van Belle, G. T., \& Thompson, R. R. 1998, AJ, 116, 981

Gehrz, R. D., Hackwell, J. A., \& Jones, T. W. 1974, ApJ, 191, 675

Glebocki, R., Gnacinski, P., \& Stawikowski, A. 2000, Acta Astron., 50, 509

Glindemann, A., Abuter, R., Carbognani, F., et al. 2000, SPIE, 4006, 2

Glindemann, A., Albertsen, M., Avila, G., et al. 2004, SPIE, 5491, 447

Jackson, S., MacGregor, K. B., \& Skumanich, A. 2004, ApJ, 606, 1196

Kervella, P., Coudé du Foresto, V., Glindemann, A., \& Hofmann, R. 2000, SPIE, 4006, 31

Kervella, P., Gitton, Ph., Ségransan, D., et al. 2003, SPIE, 4838, 858

Kervella, P., Ségransan, D., \& Coudé du Foresto, V. 2004a, A\&A, 425, 1171

Kervella, P., Thévenin, F., Di Folco, E., \& Ségransan, D. 2004b, A\&A, 426, 297

Lebouquin, J.-B., Rousselet-Perraut, K., Kern, P., et al. 2004, A\&A, 424, 719

McAlister, H. A., ten Brummelaar, T. A., Gies, D. R, et al. 2005, ApJ, 628, 439

Meilland, A., Stee, Ph., Vannier, M., et al. 2006, A\&A, submitted

Ohishi, N., Nordgren, T. E., \& Hutter, D. J. 2004, ApJ, 612, 463

Peters, G. J. 1982, ApJ, 253, L33

Petrov, R. G., \& Amber Consortium, The 2003, EAS Publications Series, Observing with the VLTI, ed. G. Perrin, \& F. Malbet, 6, 111

Quirrenbach, A., Bjorkman, K. S., Bjorkman, J. E., et al. 1997, ApJ, 479, 477

Richichi, A., \& Percheron, I. 2005, A\&A, 434, 1201

Ségransan, D., Forveille, T., Millan-Gabet, C. P. R., \& Traub, W. A. 1999, in ASP Conf. Ser., 194, 290

Slettebak, A. 1982, ApJS, 50, 55

Stee, Ph., de Araujo, F. X., Vakili, F., et al. 1995, A\&A, 300, 219

Stee, Ph., Vakili, F., Bonneau, D., \& Mourard, D. 1998, A\&A, 332, 268

Snow, T. P. 1981, ApJ, 251, 139

Tycner, C., Lester, J. B., Hajian, A. R., et al. 2005, ApJ, 624, 359

Van Boekel, R., Kervella, P., Schöller, M., et al. 2003, A\&A, 410, 37

Vinicius, M. M. F., Zorec, J., Leister, N. V., \& Levenhagen, R. S. 2006, A\&A, 446, 643

von Zeipel, H. 1924, MNRAS 84, 665

Waters, L. B. F. M. 1986, A\&A, 162, 121

Waters, L. B. F. M., Cote, J., \& Lamers, H. J. G. L. M. 1987, A\&A, 185, 206 